of perceived severity of fatigue ( $47 \%$ of variance): limiting behaviour $(\beta=0.52$, $p<0.01)$, TTB $(\beta=0.31, p<0.05)$, Depression $(\beta=0.29, p<0.05)$.

Conclusions: These correlational findings suggest that self-regulation through limiting activity should be given priority attention in cognitive behavioural interventions aimed at reducing subjective fatigue.

References:

[1] Nicassio et al. Pain 2002;100:271-9.

[2] Marques et al. Int J Behav Med 2013;20:229-38.

[3] Wolfe et al. Arthritis Rheum 1990.

[4] Wolfe et al. Arthritis Care Res (Hoboken) 2010;62:600-10.

[5] Derogatis et al. Pearson; 1993.

[6] Beurskens et al. Occup Environ Med 2000;57:353-7.

[7] Violani et al. Brain Res Bull 2004;63:415-21.

[8] Spence et al. Psychol Med 2005;35:583-93.

Disclosure of Interest: None declared

DOI: 10.1136/annrheumdis-2017-eular.5762

\section{AB0925 GENDER DIFFERENCE IN FIBROMYALGIA: COMPARISON BETWEEN MALE AND FEMALE PATIENTS FROM AN ITALIAN MONOCENTRIC COHORT}

M.P. Guzzo ${ }^{1}$, C. Iannuccelli ${ }^{1}$, M.C. Gerardi ${ }^{2}$, B. Lucchino ${ }^{1}$, G. Valesini ${ }^{1}$, M. Di Franco ${ }^{1}$. ${ }^{1}$ Dipartimento di Medicina Interna e Specialità Mediche, Sapienza Università di Roma, Rome; ${ }^{2}$ Unità di Reumatologia, ASST-Fatebenefratelli Sacco - Polo Universitario, Milan, Italy

Background: Fibromyalgia (FM) is one of the main causes of chronic widespread pain (CWP). It is characterized by CWP, which is the cardinal symptom, and the presence of more than 11 tender points (hyperalgesia). [1] Other symptoms such as fatigue, sleep disturbances, difficulties with memory and concentration, irritable bowel syndrome, headache, depression are frequent. Worldwide mean prevalence of FM is $2,7 \%$ with female/male ratio of $4: 1$. In Italy the prevalence is $3,7 \%$ with the same female/male ratio. [2]

Objectives: The aim of the study is to analyze clinical features of a cohort of male patients with CWP and to evaluate gender differences in patients diagnosed as FM.

Methods: The study population consisted of 101 consecutive male subjects referred to the Clinic for the Diagnosis and Therapy of Fibromyalgia, in the period between January 2007 and October 2016, matched with a control group of 101 females with CWP referred to the clinic in the same period. Complete clinical evaluation was performed in all patients.

Results: Ninety-seven male subjects $(96 \%)$ were referred to the clinic for a history of musculoskeletal pain, among these $53 \%$ reported fatigue and $60 \%$ complained about sleep disorders. A stressful trigger (work or family problems, bereavement, infections) at the onset of pain was reported by $42 \%$ of patients. Fifty-two percent of patients reported mood changes. Physical examination showed hyperalgesia in $15 \%$ of the subjects and a mean tender points' (TP) count was 4.6 (range $0-18$ ). The diagnosis of FM was performed according to ACR 1990 criteria, since the enrollment included patients referred to the clinic before the publication of the latest ACR criteria [3, 4]. Only 18 male subjects $(18 \%)$ fulfilled the classifying criteria. In the female group CWP was reported by $97 \%$, fatigue by $65 \%$, and sleep disorders by $72 \%$ of patients. Stressful events were reported by the $50 \%$ of female population. Mood changes were described by $54 \%$ of female subjects and were predominantly depressive. Physical examination revealed hyperalgesia in 48 subjects $(47 \%)$ and the mean TP count was 11 (range $0-18$ ). The diagnosis of FM was confirmed in $60 \%$ of subjects. Comparing the two cohorts of patients with FM, mean age at the time of the visit and mean age of onset of symptoms resulted significantly higher in females than in males $(\mathrm{p}=0.02$ and $\mathrm{p}=0.04$ respectively). There was no statistically significant difference in the number of TP, in fatigue, sleep and mood disorders and in the percentage of stressors considered as a trigger for the disease. Hyperalgesia was the only feature more common in females than in males $(p=0.03)$.

Conclusions: The significant higher frequency of hyperalgesia in women suggests a different presentation of CPW, that is the cornerstone of FM, in the two sexes. Moreover, the prevalence of FM was higher in females than in males when 1990 ACR criteria were used. It can be assumed that further gender differences could be showed applying 2010 ACR criteria.

References:

[1] Wolfe et al,1990.

[2] Queiroz LP, 2013

[3] Wolfe et al, 2010

[4] Wolfe et al, 2011

Disclosure of Interest: None declared

DOI: 10.1136/annrheumdis-2017-eular.4839

\section{AB0926 AUTONOMIC DYSFUNCTION IN FIBROMYALGIA MAY BE MEDIATED BY HYPERMOBILITY SYNDROME}

D. Aslanyan ${ }^{1}$, Z. Cipinova ${ }^{2}$, N.A. Harrison ${ }^{1}$, H.D. Critchley ${ }^{1}$, K.A. Davies ${ }^{1}$, J.A. Eccles ${ }^{1} .{ }^{1}$ BSMS; ${ }^{2}$ BSUH, Brighton, United Kingdom

Background: Neuropsychiatric symptoms are common in Fibromyalgia (FMS). FMS is associated with dysautonomia, particularly orthostatic intolerance, in which there is a phenomenological overlap with anxiety. FMS and dysautonomia are both associated with joint hypermobility (JHS).

Objectives: To investigate whether signs and symptoms of dysautonomia in FMS are mediated by JHS.

Methods: Eighteen patients with FMS (all female; mean age 41.06 years) and 19 controls (14 female; mean age 46.42 years) were recruited. JHS was assessed by Brighton Criteria. Multi-systemic symptoms suggestive of dysautonomia were quantified using Autonomic Symptoms and Quality of Life Scale (ASQoLS). Neuropsychiatric symptoms were formally quantified including anxiety level (BAI), depressive symptom level (BDI), panic disorder symptom severity (PDSS) and dissociative experiences (DES). All participants underwent autonomic function testing (9 minute tilt table with heart rate (HR) recording).

Statistical comparison between groups was performed using independent samples $t$ test and chi squared as required, correlations were explored using pearson or spearman rho as appropriate. Formal mediation analysis was performed using the method of Baron and Kenny (1), which stipulates that a mediator variable must reduce the statistical relationship between the independent and dependent variable.

Results: FMS patients had significant objective features of dysautonomia including higher baseline HR $(p=.001)$ and maximal HR during tilt $(p=.002)$ compared to controls and reported significantly higher autonomic symptom burden $(p=.001)$. Symptoms correlated with changes in physiology during autonomic challenge in patients $(r=.536, p=.039)$, but not controls. Across the study anxiety score correlated with absolute change in pre-tilt and average HR during tilt $(r=.483, p=.050)$ and symptoms of dysautonomia $(r=.813, p=.001)$. Dysautonomia symptoms correlated with DES $(r=.793, p=.001)$, PDSS $(r=.742$, $\mathrm{p}=.001)$, interoceptive sensibility $(\mathrm{r}=.627, \mathrm{p}=.007)$ and BDI $(\mathrm{r}=.502, \mathrm{p}=.040)$. There was a significant association between JHS and FMS ( $p=.001)$, but not generalized joint laxity. Across all participants, dysautonomia symptoms correlated with Beighton score $(r=.353 \mathrm{p}=.032)$ and JHS participants reported higher heart rates during autonomic challenge $(p=.002)$ and greater symptom burden $(p=.001)$. The significant relationship between autonomic symptoms and maximum HR during tilt was fully mediated by presence of JHS or FMS (Figure 1). JHS partially mediated the relationship between FMS and increased anxiety scores and fully mediated the relationship between FMS and maximum HR during tilt (Figure 2).
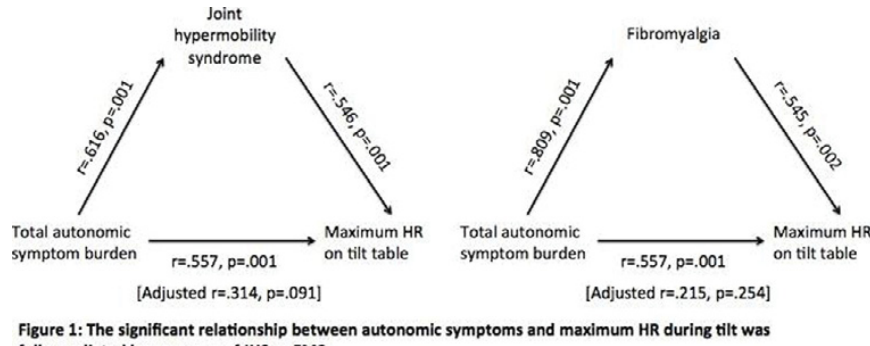

fully mediated by presence of JHS or FMS


Figure 2: IHS pattally medlited the relationship between FMS and increased anxiety scores and fully

mediated the relationship between FMS and maximum HR during tilt

Conclusions: This study confirms that objective and subjective measures of dysautonomia are more common in FMS, and associated with JHS in this cohort, suggesting a common underlying mechanism which requires further exploration. We highlight the importance of assessing patients with FMS for both JHS and dysautonomia, which may inform further management of this often challenging condition

References:

[1] Baron RM, Kenny DA. The moderator-mediator variable distinction in social psychological research: conceptual, strategic, and statistical considerations. J Pers Soc Psychol. 1986 Dec;51(6):1173-82.

Acknowledgements: This work was supported by a MRC CRTF to JAE and supported by BSUH NHS Trust NIHR Clinical Research Facility.

Disclosure of Interest: None declared

DOI: 10.1136/annrheumdis-2017-eular.5048 